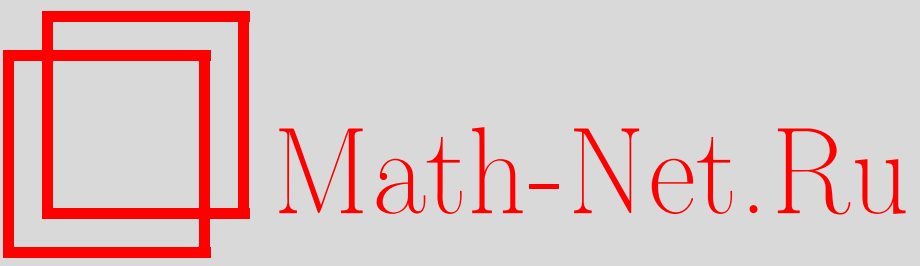

А. Э. Гутерман, Линейные отображения, сохраняющие определитель Дьедонне над произвольным телом, УМH, 2002, том 57, выпуск 4, 171-172

DOI: https://doi.org/10.4213/rm539

Использование Общероссийского математического портала Math-Net.Ru подразумевает, что вы прочитали и согласны с пользовательским соглашением

http://www.mathnet.ru/rus/agreement

Параметры загрузки:

IP: 54.81 .137 .203

26 апреля 2023 г., 14:23:58 


\title{
ЛИНЕЙНЫЕ ОТОБРАЖЕНИЯ, СОХРАНЯЮЩИЕ ОПРЕДЕЛИТЕЛЬ ДЬЕДОННЕ НАД ПРОИЗВОЛЬНЫМ ТЕЛОМ
}

\author{
А.Э. ГУТЕРМАН
}

Задача классификации линейных отображений, сохраняющих матричные инварианты, восходит к работе Георга Фробениуса [1], где получена характеризация линейных биективных преобразований пространства матриц над полем комплексных чисел, сохраняющих определитель. На протяжении прошедшего века эта теория интенсивно развивалась. В последние годы были получены существенные продвижения для матриц над коммутативньми колшцами, см. [2]. В случае некоммутативного кольца коэффициентов полулинейные отображения матриц над полупростыми артиновыми кольцами, конечно порожденными как модули над коммутативными колшцами, содержащими более двух элементов, сохраняющие вырожденность, были охарактеризованы Уонгом в [3]. Однако вопрос о некоммутативном аналоге теоремы Фробениуса (задача классификации полулинейных отображений, сохраняющих определитель Дьедонне, см. [4]) оставался открытым даже для матриц над телами. В работах [5], [6] были классифицированы полулинейные отображения матриц над телами и локальными колцами, удовлетворяющими некоторьм техническим условиям, сохраняющие определитель Дьедонне. Целью настоящей заметки является решение указанной проблемы для матриц с коэффициентами из произвольного тела.

Пусть $D$ - некоммутативное тело, являющееся алгеброй над некоторым полем $K$, множество $K \cdot 1_{D}$ содержит более двух различных элементов, $M_{n}(D)$ - множество $(n \times n)$-матриц с коэффициентами из тела $D$.

ОПредЕЛЕниЕ. Для произвольных матриц $A, B \in M_{n}(D)$ обозначим

$$
l(A, B)=\{\lambda \in K \mid \lambda A+B-\text { вырожденная матрица }\} .
$$

Лемма 1. Пусть матрица $A \in M_{n}(D), \operatorname{rk}(A) \leqslant 1$. Тогда для произвольной матрицы $B \in M_{n}(D)$ или $l(A, B)=K$, или $\left|l(A, B) \cdot 1_{D}\right| \leqslant 1$.

Для доказательства рассматривается произвольная матрица $B \in M_{n}(D)$ и предполагается, что $\left|l(A, B) \cdot 1_{D}\right|>1$, т.е. существуют различные элементы $\lambda_{1}, \lambda_{2} \in l(A, B)$. Тогда этим элементам отвечают элементы $0 \neq v_{i} \in \operatorname{Ker}\left(\lambda_{i} A+B\right), i=1,2$. Если $v_{i} \in \operatorname{Ker}(A), i=1$, 2 , то из линейности следует, что $v_{i} \in \operatorname{Ker}(B)$. Тогда $(\lambda A+B) v_{i}=0$ для всех $\lambda \in K$ и $l(A, B)=K$. Если $A v_{1} \neq 0$ и $A v_{2} \neq 0$, то $\operatorname{rk}(A)=1$. Тогда существует $0 \neq \mu \in D$ такой, что $A v_{2}=\left(A v_{1}\right) \mu$. Для произвольного элемента $\lambda \in K$ рассматривается элемент $v_{\lambda}$, определяемьй следующим образом: $v_{\lambda}=v_{1} \mu\left(\lambda-\lambda_{2}\right)+v_{2}\left(\lambda_{1}-\lambda\right)$. Непосредственная проверка показывает, что $v_{\lambda} \in$ $\operatorname{Ker}(\lambda A+B)$. Следовательно, $l(A, B)=K$.

Лемма 2. Для произвольной матрицы $A \in M_{n}(D)$ такой, что $\operatorname{rk}(A)>1$, существует такая матрица $B \in M_{n}(D)$, ито $l(A, B) \cdot 1_{D}=\left\{0_{D}, 1_{D}\right\}$.

Для доказательства матрица $A$ рассматривается как линейный оператор, действующий на пространстве столбцов длины $n$ элементов из $D$. Тогда $D^{n} \cong \operatorname{Im}(A) \oplus W \cong U \oplus \operatorname{Ker}(A)$, $\operatorname{dim}(U)=\operatorname{dim}(\operatorname{Im}(A))=\operatorname{rk}(A)>1$. Фиксируется вектор $0 \neq u_{1} \in U$ и рассматривается разложение $U_{1}=u_{1} D, U=U_{1} \oplus U_{2}$. Тогда $U_{2} \neq\{0\}$. Определяются операторы $B_{1}: U \rightarrow$ $\operatorname{Im}(A)$ через $B_{1} u_{1}=-A u_{1},\left.B_{1}\right|_{U_{2}}=0$ и $B_{2}: \operatorname{Ker}(A) \rightarrow W-$ произвольный изоморфизм векторных пространств. Непосредственная проверка показьвает, что матрица оператора $B=$ $B_{1} \oplus B_{2}: D^{n} \rightarrow D^{n}$ удовлетворяет всем требуемым условиям.

Непосредственным следствием лемм 1 и 2 является следующая характеризация матриц ранга 1 .

Работа выполнена при частичной поддержке Российского фонда фундаментальных исследований. 
СлеДСтвИе 1. Матрица $A \in M_{n}(D)$ имеет ранг, не больший единиць, тогда и только тогда, когда для любой матричьъ $B \in M_{n}(D)$ или $l(A, B)=K$, или $\left|l(A, B) \cdot 1_{D}\right| \leqslant 1$.

В силу того, что для биективного $\sigma$-полулинейного отображения $T$ справедливо $l(T(A), T(B))$ $=(l(A, B))^{\sigma}$, получим

СлеДСТвиЕ 2. Пусть $T: M_{n}(D) \rightarrow M_{n}(D)$ - биективное, $\sigma$-полулинейное над $K$ отображение, взаимно однозначное на множестве вырожденных матрич. Тогда отображение $T$ взаимно однозначно на мно жестве матриц ранга 1.

Применение теоремы Хуа, см. [7], в которой охарактеризованы биективные аддитивные взаимно однозначные на множестве матриц ранга 1 отображения матриц с коэффицииентами из тела, позволяет доказать следующие теоремы.

Теорема 1. Пусть биективное, б-полулинейное над $K$ отображсение $T: M_{n}(D) \rightarrow$ $M_{n}(D)$ взаимно однозначно на множестве вырожденных матрич. Тогда существуют такие обратимье матриць $P, Q \in M_{n}(D)$ и $\sigma$-полулинейный над $K$ автоморфизм $\mu$ тела $D$, что $T(X)=P X^{\mu} Q$ для всех $X \in M_{n}(D)$ или $T(X)=P\left({ }^{t} X^{\mu}\right) Q$ для всех $X \in M_{n}(D)$, причем матрицы $P$ и $Q$ определень единственным, с точностью до обратимого скалярного множителя, образом, $\mu$ - единственен с точностью до внутреннего автоморфизма тела $D$, здесь через ${ }^{t} X$ обозначена транспонированная матрица $\kappa$ матриче $X$.

ТЕОРема 2. Пусть сюрбективное $\sigma$-полулинейное над $K$ отображение $T: M_{n}(D) \rightarrow$ $M_{n}(D)$ сохраняет определитель Дьедонне. Тогда существуют такие матрицы $P, Q \in$ $M_{n}(D), \operatorname{det}(P Q)=\overline{1}$, и $\sigma$-полулинейный над $K$ автоморфизм $\mu$ тела $D$, что $T(X)=$ $P X^{\mu} Q$ для всех $X \in M_{n}(D)$ или $T(X)=P\left({ }^{t} X^{\mu}\right) Q$ для всех $X \in M_{n}(D)$, причем матрицы $P$ и $Q$ определены единственным, с точностью до обратимого скалярного множителя, образом, $\mu$-единственен с точностью до внутреннего автоморфизма тела $D$.

\section{СПИСОК ЛИТЕРАТУРЫ}

[1] G. Frobenius // Sitzungsber. Preuß. Akad. Wiss. Berlin. 1897. P. 994-1015. [2] L. B. Beasley, C.-K. Li, S. Pierce // Linear and Multilinear Algebra. 1992. V. 33. № 1-2. P. 109-119. [3] W. J. Wong // J. Algebra. 1988. V. 113. № 2. P. 263-293. [4] J. Dieudonné // Bull. Soc. Math. France. 1943. V. 71. P. 27-45. [5] А. Э. Гутерман, Е. М. Крейнес, А. В. Михалев // Матем. методы и прил. 1997. Т. 5. С. 119-132. [6] A. Guterman // Linear and Multilinear Algebra. 2001. V. 48. № 4. P. 293-311. [7] L. K. Hua // J. Chinese Math. Soc. (N.S.). 1951. V. 1. P. $110-163$. 